\title{
Geleitwort
}

\section{für die neue Schriftenreihe des Kolonial-Instituts der Hansischen Universität}

Das alte Hamburger Kolonial-Institut hat in den Jahren von 1908 bis 1919 auf den Gebieten der Forschung und der Lehre eine vielseitige und weitwirkende Tätigkeit entwickelt. Zeugnis dafür bildet auch heute noch die stattliche Reihe von Veröffentlichungen, die unter dem Titel: „A bhandlungen des Hamburgischen Kolonialinstituts" (Bd. I bis XXXXIII) erschienen sind. Mit der Erweiterung des alten Kolonial-Instituts zur Volluniversität im Jahre 1919 wurde diese Reihe unter dem Namen „Hansische Universität - Abhandlungen aus dem Gebiet der Auslandskunde" fortgesetzt; es sind bisher 51 Bände erschienen.

Der deutsche Wiederaufstieg und die bevorstehende Neuordnung des europäischen und afrikanischen Raumes hat auch der kolonialwissenschaftlichen Arbeit in Hamburg neue Antriebe gebracht. So wurde es möglich, das alte Hamburger Kolonial-Institut in neuer Form im Rahmen der Universität wieder erstehen zu lassen. Es wurden sämtliche Seminare, Institute oder deren Abteilungen, welche sich kolonialen Forschungen und kolonialem Unterricht widmen, mit ihren Professoren, Dozenten und Assistenten in dem erneuerten ,Kolonial-Institut" zu gemeinschaftlicher Arbeit zusammengeschlossen.

Entsprechend dieser neuen Verbindung der kolonialen Wissenschaften aller Fakultäten in einer Institution wurde in den „Abhandlungen aus dem Gebiet der Auslandskunde", die die Hansische Universität nach wie vor herausbringt, zunächst eine besondere Unterreihe gebildet mit der Bezeichnung "Schriften des Kolonial-Instituts der Hansischen Universität". Bisher sind in dieser Unterreihe 3 Veröffentlichungen erschienen. Diese Unterreihe soll weiter geführt werden mit der abgeänderten Bezeichnung: "Forschungen des Kolonial-Instituts".

Bei der zunehmenden Bedeutung, welche der kolonialwissenschaftlichen Tätigkeit in der Vorbereitung einer neuen deutschen Kolonialpolitik zukommt, vollzieht sich eine solche Erweiterung und Besonderung der kolonialwissenschaftlichen Forschung, daß eine neue Form der Veröffentlichungen gefunden werden mußte.

Das Kolonial-Institut hat sich deshalb entschlossen, neben den genannten "Forschungen des Kolonial-Instituts" eine besondere Schriftenfolge unter dem Namen "Schriften des Kolonial-Instituts der Hansischen Universität" herauszubringen. Diese Schriftenfolge wird nach dem 
inneren Zusammenhang in mehrere Sonderreihen gruppiert werden; es ist beabsichtigt, mit folgenden Reihen zu beginnen:

Kolonialwirtschaftliche Reihe,

Landeskundliche Reihe,

Völkerkundliche Reihe,

Erziehungswissenschaftliche Reihe,

Kolonialrechtliche Reihe,

Kolonialgeschichtliche Reihe.

Je nach Bedarf werden andere Reihen angeschlossen werden.

Es ist der Wunsch aller Mitglieder und Mitarbeiter des Kolonial-Instituts, daß mit diesen neuen „Schriften des Kolonial-Instituts“ nützliche Arbeit geleistet werde für die neue deutsche Kolonialpolitik, der wir entgegengehen. Der große europäische Auftrag, der vom Schicksal den Deutschen erteilt worden ist, fordert in den neuen Weltverhältnissen den vollen Einsatz auch der deutschen Wissenschaft. Die altbewährte Hamburger Kolonialwissenschaft, die auf hansischem Boden gewachsen ist, möchte auch mit dieser neuen Schriftenreihe das ihre beitragen zu dem großen gemeinsamen Werk.

$\begin{array}{cc}\text { Ad olf R ein } \\ \text { Im Dezember 1940. } & \begin{array}{c}\text { Direktor des Kolonial-Instituts } \\ \text { der Hansischen Universität }\end{array}\end{array}$

\section{Vorwort zur Kolonialwirtschaftlichen Reihe}

Die Kolonialwirtschaftslehre gehört zu denjenigen wissenschaftlichen Fachgebieten, die bisher nur in geringem Umfange gepflegt wurden. Die Lücke, die hier in der kolonialen Forschung geblieben ist, macht sich um so störender bemerkbar, je stärker das Bestreben wird, der europäischen Wirtschaft den unentbehrlichen tropischen Ergänzungsraum in vollem Ausmaße zu erschließen. In der in diesem Band begonnenen kolonialwirtschaftlichen Schriftenreihe wird mit einem bestimmten Plan an die Untersuchung der gerade für eine künftige deutsche Kolonialpolitik so wichtigen wirtschaftlichen Fragen herangegangen. Es sind vor allem die verschiedenen Wirtschaftsformen, besonders die Eingeborenenkulturen, die Bevölkerungs- und Arbeiterfragen sowie die mannigfachen Probleme der Wirtschaftslenkung im weitesten Sinne des Wortes, die in Einzelstudien einer Durchforschung unterzogen werden sollen.

Die durch den Krieg bedingten besonderen Verhältnisse, vor allem die Knappheit an geeigneten Bearbeitern der oft recht spröden Materie, wird es mit sich bringen, daß der Forschungsplan in der Reihenfolge der Einzelschriften nicht immer deutlich in Erscheinung tritt. Wir hoffen jedoch die Veröffentlichungen so einrichten zu können, daß sich schon in nicht zu langer Zeit für einige wichtige Gebiete ein Gesamtbild ergibt.

Kolonialwirtschaftliche Abteilung des Sozialökonomischen Seminars der Hansischen Universität 